\title{
Enhanced oligonucleotide microarray labeling and hybridization
}

\author{
Haider Ali Syed and David W. Threadgill \\ University of North Carolina, Chapel Hill, NC, USA
}

BioTechniques 41:685-686 (December 2006)

doi 10.2144/000112290

Microarrays are a powerful tool for functional studies at the molecular level $(1,2)$. Depending on the experiment, users have a variety of commercial and homemade choices. Most commercially available microarrays come with protocols already optimized for reproducibility and sensitivity. However, despite continued reductions in the cost of microarrays, commercially available labeling kits remain high and have now become one of the major expenses for performing microarray experiments. This article offers a source for improvement to reduce the associated costs, particularly that portion for the fluorescent dyes used by all two-color microarray platforms.

We describe a protocol that has been modified for significantly reduced amounts of reagents needed for amplification, labeling, and hybridization of probes to microarrays (detailed protocol is available at www.mouselab. org). Although optimized for the widely used Agilent Technologies microarray platform, this protocol is equally suitable for use on any other two-color platform. Specifically, this protocol has been optimized for the Low RNA Input Linear Amplification kit (Agilent Technologies, Santa Clara, CA, USA) used for sample and reference RNA amplification and labeling, which is based upon a widely used linear cRNA amplification protocol (3). The protocol produces equivalent amplified and labeled RNA compared with the original Agilent Technologies protocol, but uses one-quarter of the reagents, saving an estimated $75 \%$ in reagent costs per microarray. The major savings were achieved through a 4-fold reduction in the amount of fluorescently labeled nucleotide, in addition to reductions in other reagents required to support lower concentrations of nucleotides.

Total RNA was isolated using TRIzol ${ }^{\circledR}$ (Invitrogen, Carlsbad, CA, USA) from pooled colon, liver, and mammary glands, representing three female FVB/NJ inbred mice. The RNA was further purified with an additional ethanol precipitation step and analyzed on a LabChip ${ }^{\circledR}$ using a Bioanalyzer 2100 (Agilent Technologies) for quality

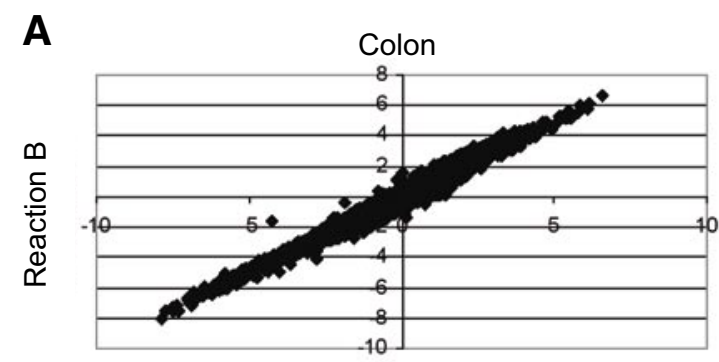

Reaction A

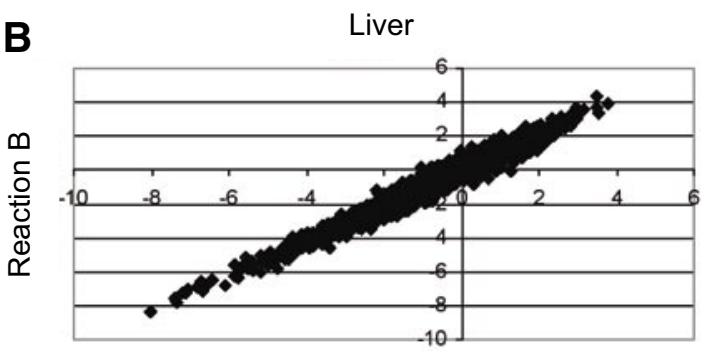

Reaction A

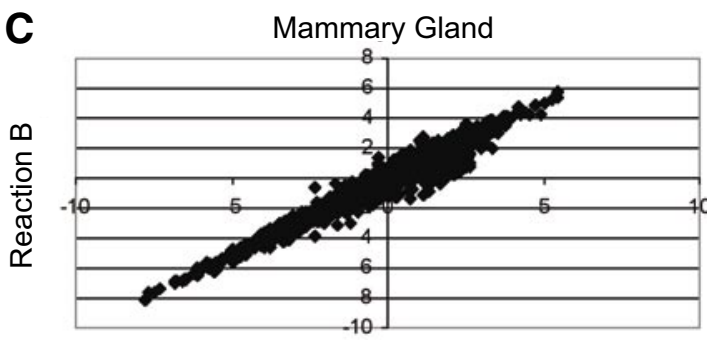

Reaction A control. The labeling and hybridization reactions were done in duplicate using the same RNA sample with the established protocol and the enhanced protocol reported here. Additional independent labeling and hybridizations were performed using the enhanced protocol to evaluate reproducibility. Mouse Universal Reference Total RNA (Stratagene, La Jolla, CA, USA) was used as the reference RNA. Reaction A was performed following the established protocol described in the Low RNA Input Linear Amplification kit, while reaction $\mathrm{B}$ was from the enhanced low reagent protocol described below. The samples from reactions A and B or from two independent $B$ reactions were co-hybridized with the reference sample to G4121A oligonucleotide mouse microarrays using SureHyb chambers (both from Agilent Technologies). The overnight hybridization and washing steps were done according to the manufacturer, and modified protocols for their respective reactions.

For the modified protocol, 250 ng total RNA was mixed with $\mathrm{T} 7$ promoter primer (Agilent Technologies) in a total volume of $5.8 \mu \mathrm{L}$ before heating to $65^{\circ} \mathrm{C}$ for $10 \mathrm{~min}$. After placing the RNA/primer mixture on ice, $4.4 \mu \mathrm{L}$ cDNA master mix containing $2 \mu \mathrm{L} 5 \times$ first strand buffer, $1 \mu \mathrm{L} 0.1 \mathrm{M}$ dithiothreitol (DTT), $0.5 \mu \mathrm{L}$ $10 \mathrm{mM}$ dNTP mix, $0.6 \mu \mathrm{L}$ Moloney murine leukemia virus reverse transcriptase

Figure 1. Correlation plots of expression data from reactions $A$ and B. RNA from (A) colon (B) liver, and $(C)$ mammary gland was labeled and hybridized independently using the Agilent Technologies and enhanced protocols before the results were compared using scatter plots. Units are $\log _{2}$ mean expression values normalized to the Mouse Universal Reference RNA (Stratagene). 
Table 1. Pearson Correlation Comparing Labeling Protocols

\begin{tabular}{|lccc|}
\hline A & \multicolumn{3}{c|}{ Reaction A Samples } \\
\hline Reaction B Samples & Colon & Liver & Mammary Gland \\
Colon & 0.99 & 0.30 & 0.56 \\
Liver & 0.30 & 0.98 & 0.36 \\
Mammary Gland & 0.55 & 0.36 & $\mathbf{0 . 9 8}$ \\
\hline B & \multicolumn{4}{|c}{ Reaction B Samples } \\
\hline Reaction B Samples & Colon & Liver \\
Colon & $\mathbf{0 . 9 8}$ & 0.27 \\
Liver & 0.26 & $\mathbf{0 . 9 9}$ \\
Colon, liver, and mammary gland samples were processed independently for reactions A (Agilent Tech- \\
nologies) and B (enhanced). (A) Samples were compared between labeling protocols and (B) between \\
replicas of the same labeling protocol. Samples were log ${ }_{2}$ red/green (R/G) Lowess mean normalized \\
with no centering. Bolded values are correlation coefficients using independent RNA samples from (A) \\
the same tissue site or (B) the same RNA used in independent labeling reactions. \\
\hline
\end{tabular}

(MMLV RT), and $0.3 \mu \mathrm{L}$ RNaseOUT TM (Agilent Technologies) were added, the contents gently mixed, and the reaction incubated at $40^{\circ} \mathrm{C}$ for $2 \mathrm{~h}$. The reaction was stopped by heating to $65^{\circ} \mathrm{C}$ for 15 min before cooling and adding $0.5 \mu \mathrm{L}$ of either $10 \mathrm{mM}$ Cy ${ }^{\mathrm{TM}} 3-\mathrm{CTP}$ or Cy5-CTP and $14.5 \mu \mathrm{L}$ transcription master mix containing $3.83 \mu \mathrm{L}$ nuclease-free water, $5 \mu \mathrm{L} 4 \times$ transcription buffer, $2 \mu \mathrm{L}$ NTP mix, $1.6 \mu \mathrm{L} 50 \%$ polyethylene glycol (PEG), $0.12 \mu \mathrm{L}$ RNaseOUT, $0.15 \mu \mathrm{L}$ inorganic pyrophosphatase, and $0.3 \mu \mathrm{L}$ T7 RNA polymerase. The contents were gently mixed and incubated at $40^{\circ} \mathrm{C}$ for $2 \mathrm{~h}$. The resulting cRNA was purified using an RNeasy ${ }^{\circledR}$ kit (Qiagen, Valencia, CA, USA) followed by quantification of the cRNA by spectroscopy using an ND1000 spectrophotometer (NanoDrop Technologies, Wilmington, DE, USA). In a new tube, $1 \mu \mathrm{g}$ Cy3- and Cy5labeled and amplified cRNA was mixed and fragmented according to the Agilent Technologies protocol before using for hybridization to a microarray. For both protocols, the overnight hybridization time was reduced from $17 \mathrm{~h}$ recommended in the commercial protocol to $10 \mathrm{~h}$, and the final wash consisted of stabilization and drying solution diluted $1: 1(\mathrm{v} / \mathrm{v})$ in acetonitrile, which gives a more even layer on the microarrays than using the concentrated solution that is provided with the hybridization kit.

After acquiring the raw images for the 10 samples (three tissue types from reactions $\mathrm{A}$ and $\mathrm{B}$ and the two independent $\mathrm{B}$ reactions, and two tissue types from reaction B replicas) from an Agilent Microarray Scanner, the images were processed using Feature Extraction software (both from Agilent Technologies). Genes were filtered to remove those that did not show mean signal intensity greater than twice the median background value in at least $70 \%$ of the microarrays before performing correlation experiments. The $\log _{2}$ for green (Cy3) over red (Cy5) intensity was calculated, and the corresponding samples from the two different protocols or replicas of the same protocol were compared using Pearson (4) or interclass correlation (5). Pearson correlation showed a very high level of correlation $(>0.98)$ between the two labeling protocols indicating that the enhanced protocol produced near identical results to the established commercial protocol (Table 1A). Likewise, replica reactions of the enhanced protocol were highly correlated $(>0.98)$ within tissue, but showed low cross-tissue correlation $(<0.27)$ (Table 1B). Scatter plots among the samples for the three tissue types (Figure 1). We also did average linkage hierarchical clustering using cluster (6) and visualized the results in TreeView (7), which showed that the corresponding samples clustered together (data not shown).

The enhanced labeling and hybridization protocol reported here using Agilent microarrays can easily be adapted to any two-color microarray platform. The results using this protocol will be indistinguishable to established protocols, but with a substantial savings, averaging over U.S. $\$ 150$ per microarray. supported the strong positive correlation

\section{ACKNOWLEDGMENTS}

This work was supported by grants to D.W.T. (National Institutes of Health nos. CA105417, CA106991, and ES11391). Support provided by center grants (National Institutes of Health nos. DK34987, ES10126, and CA16086) was also important.

\section{COMPETING INTERESTS STATEMENT}

The authors declare no competing interests.

\section{REFERENCES}

1.Adler, A.S., M. Lin, H. Horlings, D.S. Nuyten, M.J. van de Vijver, and H.Y. Chang. 2006. Genetic regulators of largescale transcriptional signatures in cancer. Nat. Genet. 38:421-430.

2.Shyamsundar, R., Y.H. Kim, J.P. Higgins, K. Montgomery, M. Jorden, A. Sethuraman, M. van de Rijn, D. Botstein, et al. 2005. A DNA microarray survey of gene expression in normal human tissues. Genome Biol. 6:R22.

3. Van Gelder, R.N., M.E. von Zastrow, A. Yool, W.C. Demant, J.D. Barchas, and J.H. Eberwine. 1990. Amplified RNA synthesized from limited quantities of heterogeneous cDNA. Proc. Natl. Acad. Sci. USA 87:16631667

4.Lin, L.I. 1989. A concordance correlation coefficient to evaluate reproducibility. Biometrics 45:255-268.

5.Lee, J., D. Koh, and C.N. Ong. 1989 Statistical evaluation of agreement between two methods for measuring a quantitative variable. Comput. Biol. Med. 19:61-70.

6. Eisen, M.B., P.T. Spellman, P.O. Brown, and D. Botstein. 1998. Cluster analysis and display of genome-wide expression patterns. Proc. Natl. Acad. Sci. USA 95:14863-14868.

7. Eisen, M.B. and P.O. Brown. 1999. DNA arrays for analysis of gene expression. Methods Enzymol. 303:179-205.

Received 18 May 2006; accepted 31 August 2006.

Address correspondence to David Threadgill, Department of Genetics, CB\#7264, University of North Carolina, Chapel Hill, NC 27599, USA. e-mail: dwt@med.unc.edu

To purchase reprints of this article, contact: Reprints@BioTechniques.com 